\section{CpG island hypermethylation is maintained in human colorectal cancer cells after RNAi-mediated depletion of DNMT1}

Angela H Ting ${ }^{1,2}$, Kam-wing Jair ${ }^{2}$, Hiromu Suzuki², Ray-Whay Chiu Yen ${ }^{2}$, Stephen B Baylin ${ }^{1,2}$ \& Kornel E Schuebel ${ }^{2}$
The role of the primary mammalian DNA methyltransferase, DNMT1, in maintaining CPG island methylation in human colon cancer cells has recently been questioned. This controversy has arisen from discrepancies between genetic knockout and RNA interference-mediated knockdown studies. Here, we re-examined the RNA interference-based approach and found that hypermethylation of single-copy genes is maintained in cells transiently and stably depleted of DNMT1. a

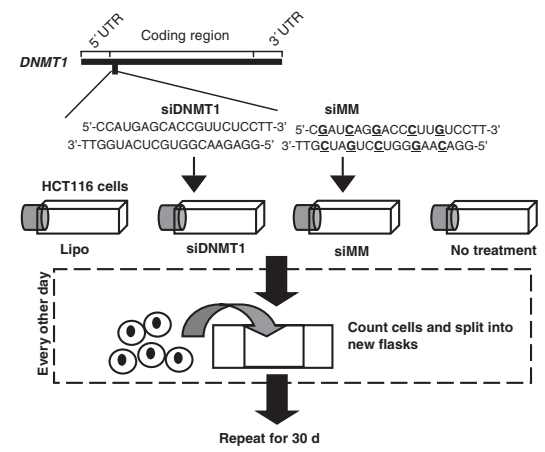

d
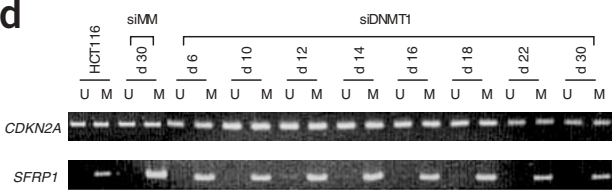

GATA4

GATA5

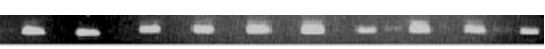

b

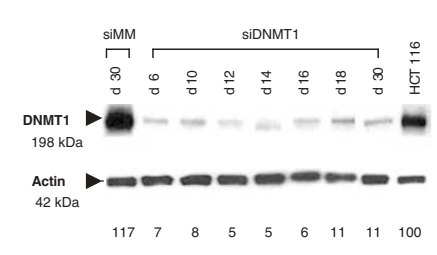

C

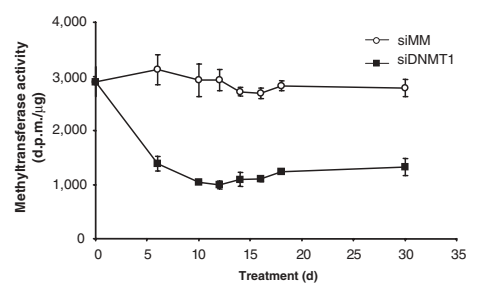

f

e

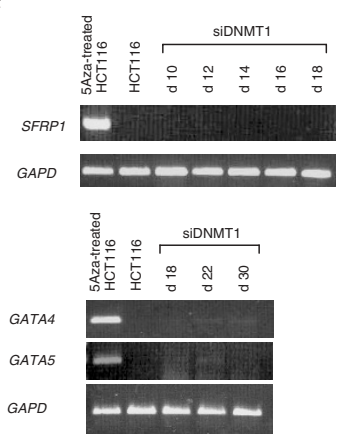

simm

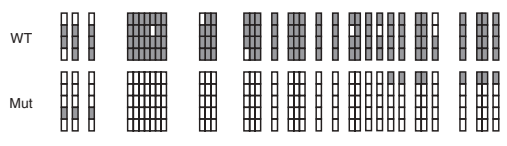

siDNMT1
d 6
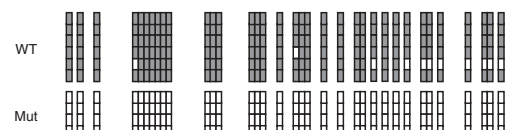

siDNMT1

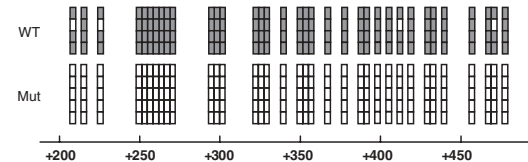

Figure 1 Transient depletion of DNMT1 in HCT116 cells after siRNA treatment. HCT116 cells (American Type Culture Collection) were cultured and transfected as previously described ${ }^{5}$. (a) Schematic outline of the siRNA treatment protocol. Duplicate cell pellets were analyzed. (b) Western-blot analysis of $5 \mu \mathrm{g}$ of cell extracts from cells treated with siRNA collected on the indicated days. The same blot was reprobed for actin to facilitate quantification. Normalized percentages of DNMT1 remaining, indicated below each lane, were calculated as follows: $100 \% \times$ (DNMT1 band intensity of sample/actin band intensity of sample) / (DNMT1 band intensity of HCT116/actin band intensity of HCT116). (c) Methyltransferase activity assays were done using $10 \mu \mathrm{g}$ of extract and $0.5 \mu \mathrm{g}$ of substrate ${ }^{3}$. Triplicate averages are shown as disintegrations per minute (d.p.m.) per $\mu \mathrm{g}$ of extract. (d) MSP analysis of CDKN2A, SFRP1, GATA4 and GATA5 promoters. The siRNA treatments are shown above the brackets. U, unmethylated; M, methylated. (e) RT-PCR analysis of SFRP1, GATA4 and GATA5 expression in cells treated with siDNMT1 and 5-aza-2'-deoxycytidine (5Aza; positive control). GAPD was included as a loading control. (f) The methylation status of individual CpGs in the CDKN2A promoter in wild-type (WT) or mutated (Mut) alleles. Filled squares, methylated; open squares, unmethylated. CpGs are shown with respect to the transcription start site.

${ }^{1}$ Program in Cellular and Molecular Medicine and ${ }^{2}$ Cancer Biology Division, The Sidney Kimmel Comprehensive Cancer Center at Johns Hopkins, The Johns Hopkins University, Baltimore, Maryland 21231, USA. Correspondence should be addressed to S.B.B. (sbaylin@jhmi.edu) or K.E.S. (kornels@jhmi.edu). 
The accumulation of 5-methylcytosine in the promoters of tumorsuppressor genes correlates with their transcriptionally silent state and is a crucial pathway for gene inactivation in human neoplasia ${ }^{1}$. The role of DNMT1 in maintaining CpG methylation in human cancer cells, extrapolated from studies done during early mouse development $^{2}$, was re-evaluated when it was determined that CpG island hypermethylation could be maintained after genetic deletion of the DNMT1 locus in the human colorectal carcinoma cell line HCT116 (ref. 3). Later experiments showed a cooperative role for the enzymes DNMT1 and DNMT3b in maintaining nearly all genomic CpG methylation ${ }^{4}$. HCT116 cells depleted of DNMT1 by treatment with antisense or small interfering RNA (siRNA) showed complete loss of $\mathrm{CpG}$ methylation in the promoter of the tumor-suppressor gene CDKN2A, leading to the conclusion that DNMT1 is essential in maintaining $\mathrm{CpG}$ methylation in these cells ${ }^{5}$.

To address this controversy, we depleted HCT116 cells of DNMT1 using the same double-stranded RNA oligonucleotides (siDNMT1) used previously ${ }^{5}$ (Fig. 1a) and included mismatched siRNA oligonucleotides (siMM) and reagent-only treatments as controls. We collected cells every $48 \mathrm{~h}$ to monitor protein amount, enzyme activity and growth (Supplementary Fig. 1 online). Quantitative western-blot analysis showed an average reduction of $92 \% \pm 3 \%$ in DNMT1 protein levels (Fig. 1b), and enzyme activity was reduced by $59 \% \pm 5 \%$ throughout the 30 -d period (Fig. 1c). These results are comparable to those obtained previously ${ }^{5}$.

We analyzed $\mathrm{CpG}$ methylation at the CDKN2A locus by methylation-specific PCR (MSP) ${ }^{6}$. This classical tumor-suppressor gene is completely inactivated in HCT116 cells by a frameshift mutation of one unmethylated allele and hypermethylation of the other allele ${ }^{7}$. We detected both methylated and unmethylated alleles in the CDKN2A promoter during the entire transfection period (Fig. 1d). We observed similar preservation of $\mathrm{CpG}$ methylation for SFRP1, GATA4 and GATA5 promoters, which are fully hypermethylated in wild-type HCT116 cells (Fig. 1d $)^{8,9}$. Corresponding retention of gene silencing for SFRP1, GATA4 and GATA5 was confirmed by RT-PCR (Fig. 1e).

We next assessed the methylation status of $35 \mathrm{CpG}$ dinucleotides in the CDKN2A promoter using the bisulfite sequencing technique ${ }^{10}$. By using primers that amplify the region spanning the frameshift mutation, we could distinguish between wild-type (methylated) and mutated (unmethylated) alleles (Supplementary Fig. 2 online). We found that $\mathrm{CpG}$ residues on the wild-type alleles were methylated at $92 \%$ of sites in cells treated with siMM and $95 \%$ of sites (on d 6) and $97 \%$ of sites (on d 14) in cells treated with siDNMT1 (Fig. 1f).

To address whether the phenotype of DNMT1 depletion in HCT116 cells is representative of colorectal cancer cell lines, we transiently knocked down expression of DNMT1 in SW480 cells (Supplementary Fig. 3 online). These cells, unlike HCT116 cells, have almost no homologous recombination and are proficient in mismatch repair but retain high transfection efficiency. Like HCT116 cells, SW480 cells depleted of DNMT1 protein and enzyme activity retained methylation and gene silencing at all of the loci that we analyzed (Supplementary Fig. 3 online).

One criticism of the genetic knockout strategy is that only the rare clones that could tolerate loss of the enzyme survived the homologous recombination and selection that was required to delete the DNMT1 locus ${ }^{5}$. To address this issue, we generated vectors a
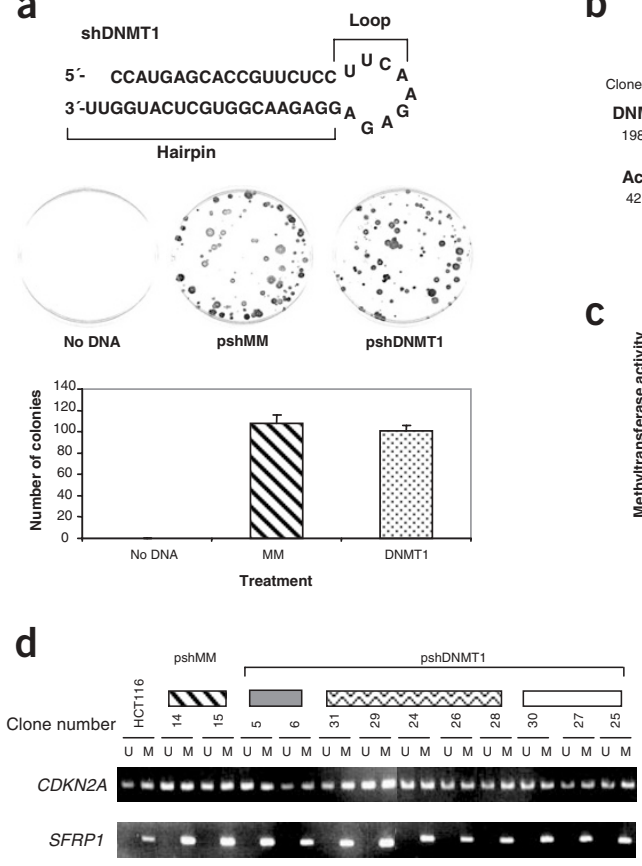

b

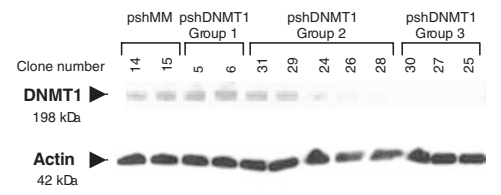

C

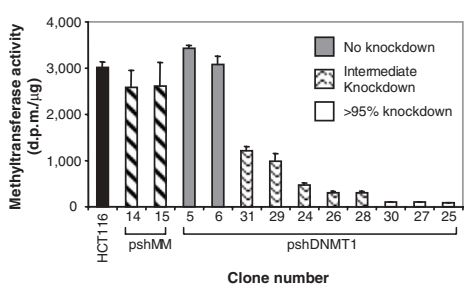

e

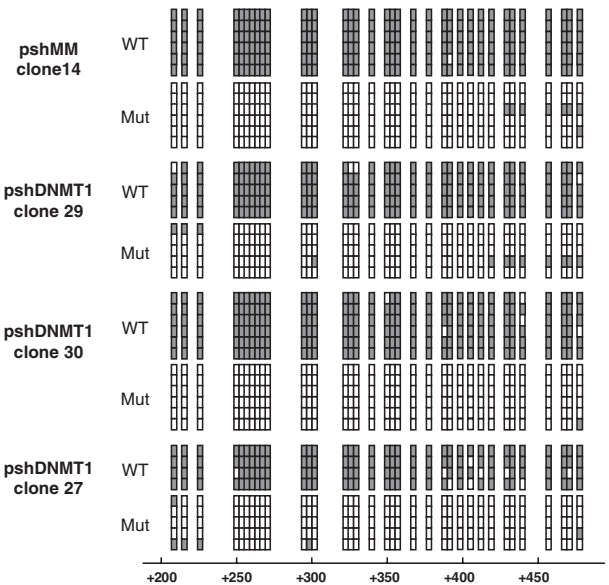

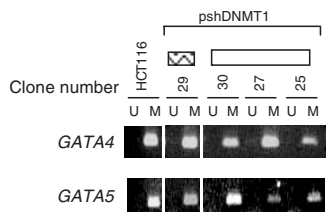

Figure 2 Generation and characterization of HCT116 cells chronically depleted of DNMT1. (a) Oligonucleotides (IDT) were cloned into pSilencer2.1 U6 Hygro (Ambion) to generate plasmids pshDNMT1 and pshMM. Transfections were done with $6 \mu \mathrm{g}$ of plasmid per 10-cm dish using Lipofectamine 2000 (Invitrogen) or with reagent alone. Selection was done in complete medium supplemented with $0.5 \mathrm{mg} \mathrm{ml}^{-1}$ of hygromycin (Sigma) for $72 \mathrm{~h}$ and maintained at $0.1 \mathrm{mg} \mathrm{ml} \mathrm{m}^{-1}$ of hygromycin for $18 \mathrm{~d}$. Triplicate dishes were stained with Giemsa on d 21 for colony counting. (b) Western-blot analysis of $5 \mu \mathrm{g}$ of cellular extracts from two pshMM and ten pshDNMT1 clones. The same blot was reprobed for actin as a loading control. (c) Methyltransferase activity assay results. pshDNMT1 cell lines are grouped according to depletion levels. (d) MSP analysis of CDKN2A, SFRP1, GATA4 and GATA5 promoters in each clone. U, unmethylated; M, methylated. (e) Bisulfite sequencing of the CDKN2A promoter in one pshMM clone (14) and three clones with intermediate (29) or severe (30 and 27) depletion of DNMT1. Filled squares, methylated; open squares, unmethylated. CpGs are shown with respect to the transcription start site. 
expressing short hairpin RNA (shRNA) species encoding the same oligonucleotide sequences as siMM (pshMM) and siDNMT1 $(\mathrm{pshDNMT} 1)^{11}$. After transfection and drug selection, we found no statistically significant differences in colony formation between the groups treated with pshMM (108 \pm 8) and pshDNMT1 (101 \pm 5 ; Fig. 2a). We randomly isolated and expanded two pshMM and ten pshDNMT1 clones for analysis. As expected, DNMT1 protein levels and enzyme activity in pshMM clones were similar to those in parental wild-type HCT116 cells (Fig. 2 b,c). The pshDNMT1 clones had one of three distinct knockdown phenotypes. The first group $(20 \%)$ showed no detectable changes in protein levels or methyltransferase activity. Most clones had a 60-90\% reduction in enzyme amount and activity, equivalent to the reductions observed in the transiently depleted cultures. Thirty percent of clones showed a $95 \%$ reduction in methyltransferase activity and an absence of protein, identical to the DNMT1-knockout cells ${ }^{3}$.

MSP analysis of cell lines with different levels of DNMT1 indicated that CpG methylation was maintained in the CDKN2A, SFRP1, GATA4 and GATA5 promoters (Fig. 2d). We further analyzed the methylation status of the CDKN2A promoter using bisulfite sequencing (Fig. 2e). The clone expressing shMM showed methylation at $>99 \%$ of the CpGs on the wild-type alleles. Clone 29 (intermediate DNMT1 knockdown) retained 98\% methylation. The clones that were severely depleted of DNMT1 showed 98\% (clone 30) and 94\% (clone 27) retention of methylation.

Our results suggest that the low frequency of DNMT1 genetic disruption does not reflect selection of rare clones but may result from well-documented differences in targeting frequencies between loci ${ }^{12}$. Stable knockdown of DNMT1 by shRNA was 30 times more efficient than genetic targeting. The difference may be due to the molecular mechanism (homologous recombination versus translation interference) underlying each strategy ${ }^{13}$.

Using both siRNA and shRNA approaches, we found that human colorectal cancer cells can maintain CpG methylation of tumor- suppressor genes in the absence of DNMT1. These findings, consistent with the phenotype of DNMT1-knockout HCT116 cells, may have important implications for the design of pharmacological interventions targeting DNA methyltransferases. Our studies suggest that inactivating DNMT1 enzyme alone may be insufficient to re-express epigenetically silenced tumor-suppressor genes and restore normal cellular growth in colorectal cancer cells. Given the compensatory functions of other DNA methyltransferases ${ }^{4}$ and the emerging importance of chromatin modifying enzymes ${ }^{14,15}$ in establishing and maintaining epigenetic gene silencing, development of combinatorial therapeutic strategies should be considered.

Note: Supplementary information is available on the Nature Genetics website.

\section{ACKNOWLEDGMENTS}

We thank B. Vogelstein and J. Herman for critical reading and B. Lee for advice, encouragement and support.

\section{COMPETING INTERESTS STATEMENT}

The authors declare competing financial interests (see the Nature Genetics website for details).

Received 6 January; accepted 21 April 2004

Published online at http://www.nature.com/naturegenetics/

1. Jones, P.A. \& Baylin, S. B. Nat. Rev. Genet. 3, 415-428 (2002).

2. Li, E. et al. Cell 69, 915-926 (1992).

3. Rhee, I. et al. Nature 404, 1003-1007 (2000)

4. Rhee, I. et al. Nature 416, 552-556 (2002).

5. Robert, M. et al. Nat. Genet. 33, 61-65 (2003).

6. Herman, J. et al. Proc. Natl. Acad. Sci. USA 93, 9821-9826 (1996).

7. Myohanen, S.K. et al. Cancer Res. 58, 591-593 (1998).

8. Suzuki, H. et al. Nat. Genet. 31, 141-149 (2002).

9. Akiyama, Y. et al. Mol. Cell. Biol. 23, 8429-8439 (2003).

10. Frommer, M. et al. Proc. Natl. Acad. Sci. USA 89,1827-1831 (1992).

11. Brummelkamp, T.R. et al. Science 296, 550-553 (2002).

12. Sedivy, J.M. et al. Science 283, 9a (1999).

13 Paddison, P.J. \& Hannon, G.J. Cancer Cell 2, 17-23 (2002).

14. Jeddeloh, J.A. et al. Nat. Genet. 22, 94-97 (1999).

15. Nan, X. et al. Nature 393, 386-389 (1998). 